\title{
Open Abdomen Management and Candida Infections: A Very Likely Link
}

\author{
Savino Occhionorelli, ${ }^{1}$ Monica Zese, ${ }^{1}$ Rosario Cultrera, ${ }^{2}$ Domenico Lacavalla, ${ }^{1}$ \\ Marco Albanese, ${ }^{1}$ and Giorgio Vasquez ${ }^{3}$ \\ ${ }^{1}$ Department of Morphology, Surgery and Experimental Medicine-University of Ferrara and Sant'Anna University Hospital of \\ Ferrara, Ferrara, Italy \\ ${ }^{2}$ Department of Medical Sciences, Centre for International Cooperation and Development, Infectious Diseases Unit-University of \\ Ferrara and Sant'Anna University Hospital of Ferrara, Ferrara, Italy \\ ${ }^{3}$ Department of Surgery, Emergency Surgery Service, Sant'Anna University Hospital, Ferrara, Italy
}

Correspondence should be addressed to Monica Zese; zsemnc@unife.it

Received 21 May 2017; Revised 16 October 2017; Accepted 17 October 2017; Published 7 December 2017

Academic Editor: Gianluca Pellino

Copyright (c) 2017 Savino Occhionorelli et al. This is an open access article distributed under the Creative Commons Attribution License, which permits unrestricted use, distribution, and reproduction in any medium, provided the original work is properly cited.

\begin{abstract}
Objective. Laparostomy can be applied in trauma, abdominal sepsis, intra-abdominal hypertension, or compartment syndrome. Systemic infections, especially if complicated by Candida, are associated with a high risk of mortality. Methods. This is a singlecentre retrospective case series of 47 cases admitted to our Department, which required laparostomy procedure; we analyzed the type of surgery, temporary abdominal closure, duration of open abdomen, complications, SOFA score, mortality with Candida infections, and empirical or targeted antifungal therapy. Results. We found that patients with Candida infection were related with a statistically significant difference $(p<0.05)$ with a complication after OA closure, total complications, time elapsed after OA application, time spent on the first surgical OA application, type of temporary abdominal closure that is used, and duration of the open abdomen. The use of empirical and targeted antifungal therapy is related to the duration of open abdomen too. Conclusions. Management of the OA is often burdened by sepsis or septic shock, especially when complicated by Candida infection. Candida score is a validated tool to identify patients who can be treated empirically, but every situation must be considered on an individual basis.
\end{abstract}

\section{Introduction}

The open abdomen $(\mathrm{OA})$ technique or laparostomy is a surgical option nowadays considered acceptable for the treatment of critically ill patients [1-3]. The key idea is to leave the abdominal cavity open in order to reduce the intra-abdominal pressure in case of abdominal hypertension and/or to allow a better control of the abdominal cavity in case of intra-abdominal infections. Abdominal contents are exposed; thus, they need to be protected with a temporary abdominal closure (TAC) [4]. Several TAC systems are used nowadays [5-8]. Usually, the role of laparostomy is closely linked to damage control surgery, especially in traumatized patients [9]. It can also be adapted in advanced sepsis or in the emergency treatment of acute peritonitis $[4,5,10-14]$, in order to prevent or control the frame of septic shock $[13,15]$. On this topic, the literature is still being debated nowadays $[3,11]$. Fascial closure can be realized $<7$ days (early) or $>7$ days (delayed) after the initial OA procedure [5]. The gold standard is the early fascial closure [5, 16-46], in order to reduce complications, but in septic patients, it is less likely to be achieved. Anyway, it should be performed as soon as possible, when abdominal sepsis is under control $[5,17-19]$. It is widely reported in the literature that the maintenance of OA predisposes patients to a further microbial and fungal contamination [20]. Therefore, antibiotic and antifungal therapies have an important role for controlling the source of sepsis and the risk of complications during and after laparostomy. Most common complications in $\mathrm{OA}$, like bleeding, anastomosis leakage, EAF appearance, or 
multiorgan failure (MOF), are linked to sepsis, and they can result in an increased mortality [5, 20, 21]. Several studies have demonstrated the role of invasive Candida species (spp.) infection in worsening sepsis or septic shock [22, 23]. Incidence of candidemia varies between 2 and 6.9 per 1000 admissions in ICU irrespective of the diagnosis of admission $[24,25]$, and it is associated with a high mortality rate, ranging from $35 \%$ to $60 \%$ [26]. The conditions of the patients can be monitored with outcome prediction models, just like the sequential organ failure assessment (SOFA) [27], in order to correlate the number of dysfunctional organs with the mortality in septic patients $[28,29]$. The study evaluates a continuative series of 47 cases treated in the Emergency Surgery Department of Sant'Anna University Hospital of Ferrara with a severe diagnosis of trauma, intestinal ischemia, bleeding, or peritonitis which required OA. It aims at considering the relation between Candida infections and diagnosis, duration of the OA, type of TAC used, complications, and outcomes. It also associates this data with microorganism infection and the use of prophylactic or targeted antifungal therapy.

\section{Methods}

In the Emergency Surgery Department of Sant'Anna University Hospital of Ferrara, 44 patients were admitted who required laparostomy (in the opinion of treating surgeon and anaesthesiologist) between February 2010 and September 2016. Among them, 3 patients required a second laparostomy, so a total number of 47 cases are taken into account. A written informed consent was obtained from every patient or from their legal delegates (at the time of intervention or during follow-up). Inclusion criteria were age $>18$ years and patients with Björck 2016 Classification System [30] grades 1a, 1b, 2a, $2 \mathrm{~b}$, and $2 \mathrm{c}$. Exclusion criteria were age $<18$ years, pregnancy, malignancy, patients with Björck 2016 Classification System grades $3 \mathrm{a}, 3 \mathrm{~b}$, and 4 . Data were collected by the computerized hospital system and medical records. Source data included preoperative parameters: age, sex, weight, height, admittance diagnosis, and comorbidities. In our Department, different kinds of TAC were used [5]: Bogotà-Bag like (we used a like sterile 3 litre saline bag cut, shaped, and sutured to fascial edges) and Opsite Sandwich technique (we covered the abdomen with a sheet of polyethylene, surrounded by Opsite, abdominal packs, two suction drains, and wall suction). This two techniques could be associated with approaching skin as in skin-only closure technique, realizing a mixed technique and VAC therapy. Opsite Sandwich technique indication, length of the OA ( $<7 />7$ days) [46], and number and distance of relooks were also considered. The study analyzed data in relation to ileus, colon, or intra-abdominal parenchymal organs (liver, pancreas, or kidney) involved. In the considered cases, the stomach was never affected. Complications have been analyzed following the Clavien-Dindo classification [31]. In our study, we have considered bleeding which required blood transfusion (grade IIa), intestinal ischemia (grade IVb), anastomosis leakage (grade IIIb), post operatory bilious fistula (grade IIIa), entero-atmospheric fistula (EAF) (grade IIIa), wound dehiscence (grade IIa), and MOF (grade
TABle 1: Comorbidities and ASA score.

\begin{tabular}{lcc}
\hline Comorbidities & $N$ & $\%$ \\
\hline Hypertension & 21 & 44.7 \\
Heart diseases & 16 & 34.0 \\
Renal diseases & 9 & 19 \\
Pneumological disorders & 3 & 6.4 \\
Previous cancer & 7 & 14.9 \\
Immune suppression & 1 & 2.1 \\
Diabetes & 7 & 14.9 \\
Obesity & 23 & 48.9 \\
ASA score & & \\
$\quad$ I & 2 & 4.3 \\
II & 7 & 14.9 \\
III & 19 & 40.4 \\
IV & 17 & 36.2 \\
V & 2 & 4.3 \\
\hline
\end{tabular}

IVb) among OA relooks and after OA definitive closure. These parameters were correlated with bacterial and Candida infections. Microorganisms were analyzed instead of detected with bronchial, urine, blood, and peritoneal analyses in every patient. Data were also related to time spent after OA application and appearance of Candida infections ( $<7 />7$ days) and antimicrobial and antifungal therapies in both ICU and Surgical Department with a follow-up of 2 months. Organ dysfunction was evaluated using the SOFA score. Every case was classified following 3 degrees of SOFA score steps in relation to mortality risk: $<3,3-9$, and $>9$. For trauma, we used the Injury Severity Score (ISS) classification [32]. Data were analyzed with statistic chi-square test, considering $p<0.05$ as statistically significant.

\section{Results}

The study included 47 cases of OA performed in 44 patients. Three patients, all males, required a second laparostomy after the closure of the previous one: two for peritonitis and one for abdominal bleeding. Of 44 patients, 15 were females (34\%) and 29 were males (66\%) with a female: male ratio of $1: 1.9$. The average age was 63 years (median 68, range 24-86), and the average ages of women and men were 69.7 and 58.8, respectively. Average BMI was $28.7 \mathrm{~kg} / \mathrm{m}^{2}$ (range $17-47 \mathrm{~kg} / \mathrm{m}^{2}$ ). Comorbidities and ASA score [33] are shown in Table 1 . We admitted $27 / 44$ patients from triage; $17 / 44$ patients were already hospitalized in other Hospital Departments (Table 2). All cases have been analyzed considering ileal, colonic, or other intra-abdominal parenchymal organ involvement (Table 2). The 6 traumatized patients were evaluated in triage using the ISS score: the average value was 28.8 (range $21-38$, median 27 ). The study of statistical correlation showed the presence of a significant relation between the concerned intestinal tract and the application of OA $(p<0.05)$. OA was applied to prevent IAH in $21 / 47$ cases $(44.7 \%)$, while it was performed in the presence of IAH in the other cases $26 / 47$ (55.3\%) [34]. The 
TABLE 2: Cases admitted, diagnosis, and abdominal involvement.

\begin{tabular}{lccc}
\hline Diagnosis & $\begin{array}{c}\text { Trauma/intestinal } \\
\text { bleeding }\end{array}$ & Ischaemia & Peritonitis \\
\hline Triage & $6(12.76 \%)$ & $9(19.14 \%)$ & $12(25.6 \%)$ \\
Already hospitalized & $5(10.6 \%)$ & $7(14.9 \%)$ & $8(17 \%)$ \\
Total & $11(23.4 \%)$ & $16(34 \%)$ & $20(42.6 \%)$ \\
Intestinal involvement & & & \\
Ileus & $4(8.5 \%)$ & $11(23.4 \%)$ & $3(6.4 \%)$ \\
Colon & $3(6.4 \%)$ & $5(10.6 \%)$ & $10(21.3 \%)$ \\
Liver, spleen, kidney & $3(6.4 \%)$ & $0(0 \%)$ & $5(10.6 \%)$ \\
Reinterventions & $1(2.1 \%)$ & $0(0 \%)$ & $2(4.2 \%)$ \\
\hline
\end{tabular}

SOFA score was $<3$ in $8 / 47$ cases (17\%), $3-9$ in $15 / 47$ (31.9\%), and $>3$ in the other $24 / 47$ cases (51.1\%), so in $83 \%$ of cases, there was a high risk of mortality related to sepsis. We also evaluated the duration of the OA maintaining ( $<7$ or $>7$ days) $[5,17]$ and the type of OA used [35]. In $32 / 47$ cases $(68.1 \%)$, OA was kept $<7$ days, while only in 15/47 (31.9\%), OA was kept over 7 days. We used Bogotà-Bag like in 27/47 cases (57.4\%), Opsite Sandwich technique in $6 / 47$ cases (12.8\%), and mixed technique (Bogotà-Bag like or Opsite Sandwich technique + skin-only closure) in $12 / 47$ cases (25.5\%). VAC therapy was used only twice $(4.3 \%)$. Considering the 3 patients treated after a previous laparostomy, 2 of them kept $\mathrm{OA}<7$ days and only one $>7$ days. In all 3 cases and in both laparostomies, we exploited Bogotà-Bag like TAC. Anastomosis was done in $34 / 47$ cases $(77.3 \%), 13 / 47$ at the first look $(27.66 \%)$ and $21 / 47$ at the following relooks (44.68\%). In 13/47 cases (27.66\%), a temporary or definitive stoma was created. Only $3 / 47$ cases (6.4\%) developed EAF [36]. Almost always, but 4 cases $(7.2 \%)$, abdominal wall was closed by direct closure. In 4 cases, the closure was done by a biological prosthesis; of them, 2 cases (1.8\%) also needed a component separation technique [37]. Complications appeared in 29/47 cases (61.7\%). Complications among relooks were 17/47 (36\%): abdominal hemorrhage in 8 cases (17\%), development of MOF in 6 cases (12.7\%), anastomosis leakage in 2 cases (4.2\%), and caecum ischemia in 1 case $(2.1 \%)$. Complications after OA closure appeared in $21 / 47$ cases (44.7\%): hemorrhage in 2 cases $(4.3 \%)$, anastomotic leakage in 4 cases $(8.5 \%)$, wound dehiscence in 6 cases $(12.8 \%)$, MOF in 3 cases (6.4\%), and bilious fistula in 2 cases (4.3\%). Furthermore, in 4 cases, pulmonary thromboembolism was detected and treated adequately (in Figure 1, complications are shown among relooks and after abdominal closure). Several types of bacteria were found in blood analysis in 19/47 cases $(40.4 \%)$ and in peritoneal analysis in $32 / 47$ cases $(68.1 \%)$. Sometimes, different kinds of microorganisms were found in different cultural analyses of the same case. The bacterial population was very heterogeneous and therefore not sufficient to allow for a statistically significant analysis. Principal bacteria species and their findings are shown in Table 3. Candida spp. were detected in $17 / 47$ cases (36.2\%) and in several cases, also in different analyses of the same patient

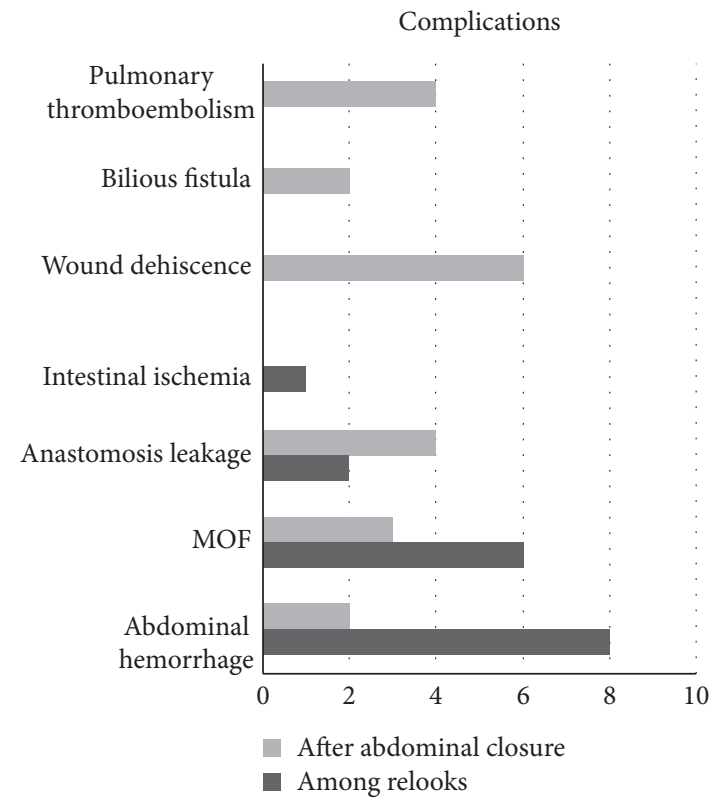

Figure 1: Complications among relooks and after abdominal closure: in the picture are evidenced principal complications among and after abdominal relooks.

(Table 4). Candida infection (revealed by blood and/or peritoneal cultures) appeared in $15 / 17$ cases $(88.2 \%)$. In the remaining $2 / 17$ cases (11.8\%), C. parapsilosis and C. albicans were evidenced in bronchial analysis only and they had not been treated with an antifungal therapy. Bronchial Candida contamination was not treated in agreement with the infectious specialist because of a single colonization. Empirical antifungal therapy was established at the moment of admission in ICU in $7 / 47$ cases (14.9\%), and only $4 / 47(8.6 \%)$ of them developed a subsequent Candida infection. In 3 of these cases, C. albicans was detected in peritoneal fluid analysis, and in the latter case, C. glabrata and C. albicans were found in peritoneal analysis and at the same time $C$. parapsilosys in urine analysis. In $11 / 47$ cases (23.4\%), a targeted therapy was applied only when cultures detected Candida spp. In our study, there was no evidence of correlation between Candida infection and the three different grades of SOFA score, near-operative death, the indication of OA application, complications among relooks, different intestinal involvements, comorbidities (such as obesity, renal pathologies, diabetes, and immune suppression), and the relation between IAH and the creation of anastomosis or stoma $(p>0.05)$ (Table 5). The overall mortality in our study is $13 / 44$ patients (29.5\%): 4 women (9.1\%) and 9 men (20.5\%), 6 of them (13.6\%) with invasive Candida infection. Overall, 6 of the 17 patients affected by Candida infections died (35.3\%). In one case, there was only bronchial Candida contamination; in 3 cases, targeted therapy was applied; and in 2 cases, patients were treated with empirical antifungal therapy. We found a relation between Candida infection and both complications after OA closure and total complications. We also considered the elapsed time after the OA application and the first positive analysis for Candida spp. ( $<7$ days or $>7$ days). We found a statistically significant relation between 
TABLE 3: Different bacteria in blood and peritoneal analysis. We considered the total where we found the presence of bacteria, and we divided them in base of blood or peritoneum detection.

\begin{tabular}{lccccc}
\hline Bacteria & E. coli & Klebsiella pneumoniae & Enteorococcus faecalis/faecium & Pseudomonas aeruginosa & Staphylococcus/streptococcus \\
\hline Total cases & $22(46.8 \%)$ & $10(21.3 \%)$ & $21(44.7 \%)$ & $7(14.9 \%)$ & $29(61.7 \%)$ \\
Peritoneum & $19(40.4 \%)$ & $9(19.1 \%)$ & $16(34 \%)$ & $5(10.6 \%)$ & $21(44.7 \%)$ \\
Blood & $10(21.3 \%)$ & $2(4.3 \%)$ & $12(25.5 \%)$ & $5(10.6 \%)$ & $16(34 \%)$ \\
\hline
\end{tabular}

Table 4: Different Candida species and their findings.

\begin{tabular}{|c|c|c|c|c|c|}
\hline Candida & parapsilosis & albicans & glabrata & mirabilis & tropicalis \\
\hline Bronchial & $1(5.9 \%)$ & $4(23.5 \%)$ & $1(5.9 \%)$ & 0 & 0 \\
\hline Urine & $1(5.9 \%)$ & $3(17.6 \%)$ & $1(5.9 \%)$ & $1(5.9 \%)$ & $1(5.9 \%)$ \\
\hline Peritoneum & 0 & $10(58.8 \%)$ & $3(17.6 \%)$ & 0 & 0 \\
\hline Blood & 0 & $2(11.8 \%)$ & 0 & 0 & 0 \\
\hline
\end{tabular}

TABle 5: Not statistically significant $(p>0.05)$ and $\%$ among Candida infection, the three different grades of SOFA score, near-operative death, the indication of OA application, complications among relooks, different intestinal involvements, and the relation between IAH and the creation of anastomosis or stoma.

\begin{tabular}{|c|c|c|c|c|c|c|}
\hline & & \multicolumn{5}{|c|}{ Candida infection } \\
\hline & & \multirow{2}{*}{$\frac{\text { Yes }}{6}$} & \multirow{2}{*}{$\frac{\text { No }}{2}$} & \multicolumn{2}{|c|}{$\%$ at total cases (47) } & \multirow[t]{2}{*}{$p>0.05$} \\
\hline \multirow{3}{*}{ Sofa score } & $<3$ & & & 2.82 & 0.94 & \\
\hline & $3-9$ & 7 & 8 & 3.29 & 3.76 & \multirow[t]{2}{*}{0.42} \\
\hline & $>9$ & 14 & 10 & 6.58 & 4.7 & \\
\hline \multirow{2}{*}{ Mortality rate } & Dead & 7 & 6 & 3.29 & 2.82 & \multirow{2}{*}{0.59} \\
\hline & Alive & 20 & 24 & 9.4 & 11.28 & \\
\hline \multirow{3}{*}{ OA indication } & Trauma bleeding & 6 & 5 & 2.82 & 2.35 & \multirow{3}{*}{0.95} \\
\hline & Ischemia & 9 & 7 & 4.23 & 3.29 & \\
\hline & Peritonits & 12 & 8 & 5.64 & 3.76 & \\
\hline \multirow{2}{*}{ Complications among relooks } & With complications & 10 & 7 & 4.7 & 3.29 & \multirow{2}{*}{0.88} \\
\hline & Without complications & 17 & 13 & 7.99 & 6.11 & \\
\hline \multirow{3}{*}{ Intestinal involvement } & Ileus & 12 & 7 & 5.64 & 3.29 & \multirow{3}{*}{0.77} \\
\hline & Colon & 10 & 8 & 4.7 & 3.76 & \\
\hline & Liver, spleen, kidney & 5 & 5 & 2.35 & 2.35 & \\
\hline \multirow{2}{*}{ IAH } & Yes & 14 & 12 & 6.58 & 5.64 & \multirow{2}{*}{0.57} \\
\hline & No & 13 & 8 & 6.11 & 3.76 & \\
\hline \multirow{2}{*}{ Anastomosis } & Yes & 19 & 15 & 8.93 & 7.05 & \multirow{2}{*}{0.72} \\
\hline & No & 8 & 5 & 3.76 & 2.35 & \\
\hline \multirow{2}{*}{ Stoma } & Yes & 8 & 3 & 3.76 & 1.41 & \multirow{2}{*}{0.24} \\
\hline & No & 19 & 7 & 8.93 & 3.29 & \\
\hline
\end{tabular}

time spent on the first surgical OA application and Candida infection appearance. There was also a statistically significant relation between Candida infection and the duration of the OA. We found a correlation between Candida and the type of TAC used. This analysis also considered the two cases of VAC therapy OA, even though they are not to be considered statistically significant. More infections occurred with the use of Bogotà-Bag like; no infections occurred with Opsite Sandwich technique and a provided equal in mixed technique (Table 6, Figure 2). However, we did find a statistically significant relation between antifungal therapy and the total duration of the OA. In particular, antifungal therapy was not used in the majority of OA which were kept $<7$ days. Data showed that Candida infection is less frequent when the duration of the $\mathrm{OA}$ is $<7$ days, as well as the setting of empirical antifungal therapy. Conversely, when $\mathrm{OA}$ is kept $>7$ days, antifungal therapy is used in more cases (Table 6, Figure 2). 
TABLE 6: Statistical significance at $p<0.05$ and \% among Candida infection and type of TAC used, duration of OA, total complications, and complications after OA closure.

(a)

\begin{tabular}{|c|c|c|c|c|c|c|}
\hline \multirow{3}{*}{ Total complications } & \multirow{3}{*}{$\begin{array}{l}\text { Case positive } \\
\text { Case negative }\end{array}$} & \multirow{3}{*}{$\begin{array}{c}\text { Yes } \\
3 \\
14\end{array}$} & \multicolumn{3}{|c|}{$\begin{array}{l}\text { Candida } \\
\text { Total cases (47) at } \\
p<0.05 \text { and } \%\end{array}$} & \multirow{3}{*}{$\begin{array}{r}p<0.5 \\
0.03\end{array}$} \\
\hline & & & 15 & 6.40 & 32 & \\
\hline & & & 15 & 29.60 & 32 & \\
\hline \multirow{2}{*}{ Complications after OA closure } & Case positive & 11 & 10 & 23.40 & 21.28 & \multirow{2}{*}{0.03} \\
\hline & Case negative & 6 & 20 & 12.77 & 42.55 & \\
\hline \multirow{2}{*}{ Duration of OA } & $<7$ days & 7 & 25 & 14.89 & 53.19 & \multirow{2}{*}{0.003} \\
\hline & $>7$ days & 10 & 5 & 21.28 & 10.64 & \\
\hline \multirow{4}{*}{ Type of TAC used } & Bogotà-Bag like & 9 & 18 & 19.15 & 38.30 & \multirow{4}{*}{0.04} \\
\hline & Opsite Sandwich technique & 0 & 6 & 0.00 & 12.77 & \\
\hline & Mixed & 6 & 6 & 12.77 & 12.77 & \\
\hline & VAC & 2 & 0 & 4.26 & 0.00 & \\
\hline
\end{tabular}

(b)

\begin{tabular}{cccccc}
\hline & & $<7$ days & $>7$ days & \multicolumn{2}{c}{$\begin{array}{c}\text { Total cases }(47) \text { at } \\
p<0.05 \text { and } \%\end{array}$} \\
\hline \multirow{3}{*}{ Duration of OA } & $<7$ days & 3 & 3 & 6.38 & 6.38 \\
& $>7$ days & 4 & 7 & 8.51 & 14.89 \\
& No infection & 25 & 5 & 53.19 & 10.64 \\
\hline
\end{tabular}

(c)

\begin{tabular}{|c|c|c|c|c|c|c|}
\hline & & & & ngth of & & \\
\hline & & $<7$ days & $>7$ days & $\begin{array}{r}\text { To } \\
p\end{array}$ & & $p<0.05$ \\
\hline & Not used & 22 & 4 & 46.81 & 8.51 & \\
\hline Antifungal & Empirical & 4 & 6 & 8.51 & 12.77 & 0.02 \\
\hline & Targeted & 6 & 5 & 12.77 & 10.64 & \\
\hline
\end{tabular}

\section{Discussion}

Many aspects must be considered when OA is used, such as the reason for application of the laparostomy, age, and comorbidities of the patient, septic status, and hydration conditions [5]. As revealed by Cristaudo et al. [36], currently, there are no published reviews of prognostic factors with regard to definitive fascial closure, mortality, and intraabdominal complications of patients being managed with OA. Intra-abdominal infections are considered as emergencies, and a successful outcome depends on early diagnosis, an appropriate surgical treatment, and adequate antibiotic therapy [47]. The control of invasive Candida infections is related to a reduced mortality [23, 38, 47]; nevertheless, criteria for starting an empirical antifungal therapy are poor. In many cases, unnecessary starting of antifungal therapy can cause resistance $[39,40]$. The colonization rate of Candida spp. reaches up to $80 \%$ in patients who reside in intensive care units (ICUs) more than a week, and the mean rate of development of invasive disease is $10 \%$ in colonized patients [48]. Candida colonization is considered multifocal when the same or two different species are found in two or more nonadjacent loci of the organism, such as urine or bronchial expectorate [23]. As reported by Leroy et al. in [23], invasive Candida infection was defined by at least one positive blood culture or peritonitis, diagnosed by macroscopic findings and direct examination or positive culture for Candida in the peritoneal fluid collected during a surgical procedure. Invasive candidemia can be defined using the criteria proposed by Leon et al. in the "Candida score" [41]. Components of the Candida score are severe sepsis, total parenteral nutrition, surgery, multifocal Candida colonization, invasive mechanical ventilation, central venous catheter, urinary catheter, antibiotic therapy $>5$ days in the last 2 weeks, renal replacement therapy, insulin-dependent diabetes, and immune suppression [23]. In our study, the SOFA score was $>3$ in $39 / 47$ cases $(83 \%)$ and total parenteral nutrition was always set at admission in ICU, just like invasive mechanical ventilation, central venous catheter, and urinary catheter. In cases where we found multifocal colonization, we considered diabetes, immune suppression, renal diseases, and previous antimicrobial therapy without finding a statistically significant relation with 


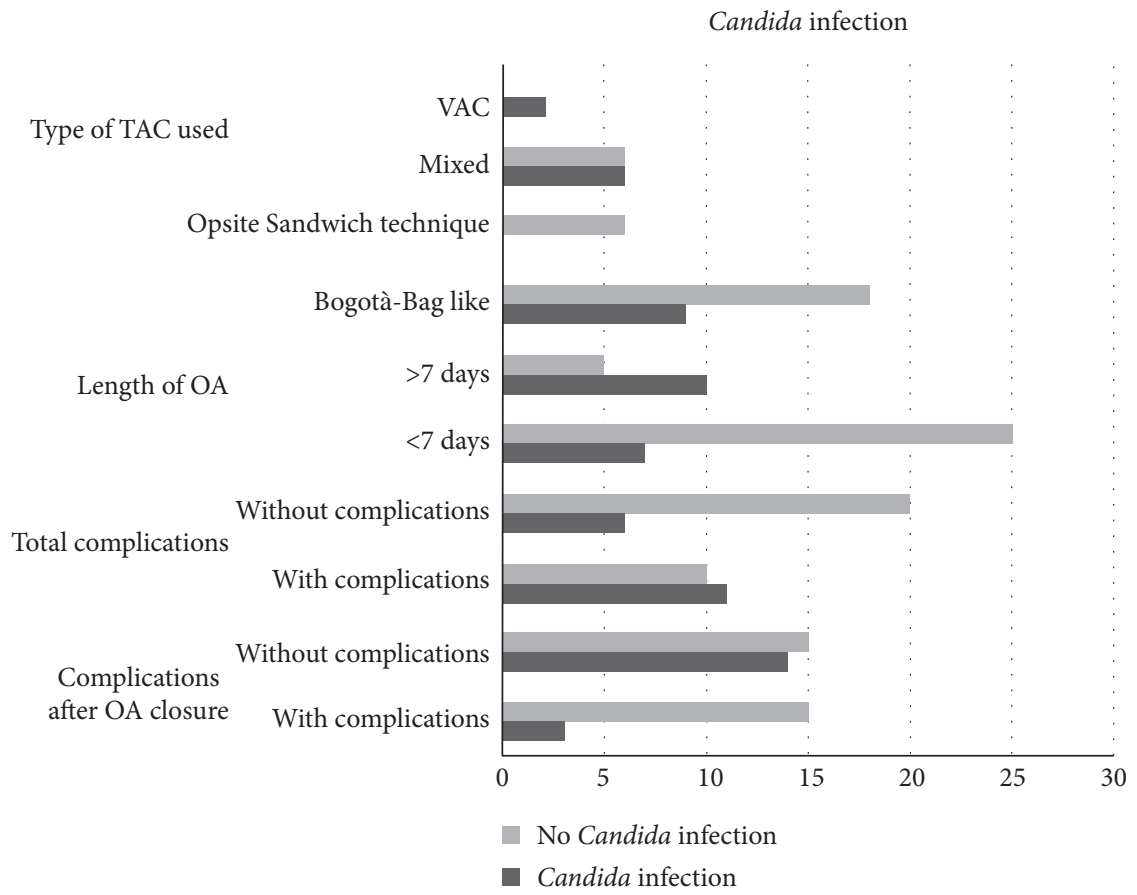

FIgure 2: Correlation among Candida infection and type of TAC used, duration of OA, total complications, and complications after OA closure.

Candida infection. Candida infection was not significantly related with immune suppression and insulin-dependent diabetes. As reported by Leroy et al. [23], criteria for starting empirical antifungal therapy in ICU patients are poorly defined and recent IDSA guidelines suggesting that "empirical antifungal therapy should be considered in critically ill patients with risk factors for invasive candidiasis and no other known cause of fever" could lead to an overuse of antifungal agents. Despite the literature is still being debated on this topic, the "Candida score" can be considered an effective tool to guide clinicians in applying antifungal therapy. In our case series, empirical therapy was set in 7 cases. In 2 cases, Candida was found in bronchial expectorate without fever or multiple colonization, so it was not treated, in agreement with the infectious specialist. Targeted therapy was applied in 11 cases after the detection of Candida spp. in peritoneum or in blood analysis. The definitive decision to empirically treat Candida is left at the single anaesthesiologist in our Department. Some studies, as [20,42], depict how microbial colonization evolves during OA maintenance. Candida infection is not an exception, and the risk of the development increases with the patient's vulnerability [43]. In our case series, we underlined that a longer OA maintenance increases the risk of developing Candida infection. In [47], it is reported that Candida peritonitis is burdened by a high mortality rate (about 38\%) and our case series attests to these values. Anyway, in our study, we must consider some biases. First of all, the small number of patients was considered, who developed Candida infection. Furthermore, there were 2 patients which required VAC therapy. However, in both cases, EAF appeared; therefore, we decided to not use this device in further cases. Secondly, our study is encumbered by the fact that during the admission, in conditions of urgency, our Department works with surgeons of different teams; hence, the decision to create OA and type of TAC is left at the discretion of the relevant surgeon. The great heterogeneity of bacteria detected in blood and/or peritoneal analysis could be indicative for sample contamination or colonization as confirmed by the poor efficacy of antibiotic therapy to resolve the post operative infective complications. Moreover, we showed the good response to antifungal therapy. On the other hand, an important bias has originated due to the possibility that the patient's vulnerability could be linked to previous or actual antibiotic therapies or concomitant infections. So we decided to evaluate only Candida infection and OA management, with the aim of understanding the presence or absence of a statistically significant relation. We are aware that our data need to be integrated into a more complex analysis system. For this reason, our Department has taken part in the IROA multicenter study $[44,45]$ and plans to realize a prospective study considering OA management and Candida infection appearance. The results of our case series showed that the use of different TACs, even Bogotà-Bag like, cannot be discouraged, confirming the results of the recent IROA multicenter study [44]. The choice of exploiting OA not only in traumatized patients, but also in peritonitis and sepsis, is a working treatment in which we believe. Our idea is also enforced by an unchanged death rate in a framework that is burdened by a high mortality rate itself [44] in a high septic risk (SOFA score in fact was $>3$ in $83 \%$ of cases). Surely, this is a case series which concerns a small number of patients in a single centre, but it can represent a starting point for a better and more standardized way of treatment in different situations regarding serious diagnosis. Statistically significant relations may 
be altered by the reduced number of cases, and our study can be considered as an initial assessment of the relation between Candida infection and the various aspects of laparostomy. However, it remains an initial study. Our follow-up deliberately analyzes short distance of time, to understand which complications can occur during near-operative time.

\section{Conclusions}

OA technique and its use in critical situations are still hotly debated in the literature. No matter how seemingly effective, the annexed life-treating complications, which require highly experience in surgical, infectious, and anesthesiology treatments, must be considered when using OA. The right choice of TAC and the early fascial closure of the abdomen appear to be essential to avoid additional risk situations. Candida infection in debilitated patients, as well as patients who are subjected to OA, implies a great risk to mortality. For this reason, the treatment of invasive Candida infection should be based on predictive models, such as the "Candida score" without, in our opinion, forgetting to consider the needs and the characteristics of every individual patient, in order to avoid over- or underestimated treatment. Of course, much remains to be done to achieve optimal results in the treatment of this critical situation.

\section{Abbreviations}

OA: Open abdomen

IAH: Intra-abdominal hypertension

ACS: Abdominal compartment syndrome

TAC: Temporary abdominal closure

SOFA: Sequential organ failure assessment score

EAF: Entero atmospheric fistula

MOF: Multiorgan failure

C.: $\quad$ Candida

spp.: Species

ISS: Injury Severity Score

ASA: American Society of Anesthesiologist Score.

\section{Additional Points}

Availability of Data and Materials. Data were collected by the computerized hospital system (SAP Front End@) and Ormaweb(C) and medical records. The datasets used and analyzed during the current study are available from the corresponding author on a reasonable request. All data generated or analyzed during this study are included in this published article.

\section{Consent}

Written informed consent was obtained from the patients or their legal delegates for the publication of this article. A copy of the written consent is available for review by the Editor-in-Chief of this journal.

\section{Conflicts of Interest}

The authors declare that they have no competing interests.

\section{Authors' Contributions}

Domenico Lacavalla and Marco Albanese with Monica Zese made the acquisition, analysis, and interpretation of the data. Marco Albanese and Giorgio Vasquez drafted and wrote the article. Giorgio Vasquez, Savino Occhionorelli, and Rosario Cultrera checked the bibliography and revised the article. All authors approved the final manuscript.

\section{Acknowledgments}

The authors thank Ilse Huijbregts, coassistant Master in Medicine Maastricht University, Faculty of Health, Medicine and Life Sciences, for language suggestions.

\section{References}

[1] A. MacLean, T. O’Keefe, and J. Augenstein, "Management strategies for the open abdomen: survey of the American Association for the surgery of trauma membership," Acta Chirurgica Belgica, vol. 108, no. 2, pp. 212-218, 2008.

[2] A. W. Kirkpatrick, K. B. Laulpand, S. Karmali et al., "Spill your guts! Perceptions of trauma association of Canada member surgeons regarding the open abdomen and the abdominal compartment syndrome," The Journal of Trauma, vol. 60, no. 2, pp. 279-286, 2006.

[3] J. Rezende-Neto, T. Rice, E. S. Abreu, O. Rotstein, and S. Rizoli, "Anatomical, physiological, and logistical indications for the open abdomen: a proposal for a new classification system," World Journal of Emergency Surgery, vol. 11, no. 1, p. 28, 2016.

[4] A. K. Leppäniemi, "Laparostomy: why and when? Leppäniemi," Critical Care, vol. 14, no. 2, p. 216, 2010.

[5] M. Sartelli, F. M. Abu-Zidan, L. Ansaloni et al., "The role of the open abdomen procedure in managing severe abdominal sepsis: WSES position paper," World Journal of Emergency Surgery, vol. 10, no. 1, p. 35, 2015.

[6] A. Esteban, F. Frutos-Vivar, N. D. Ferguson et al., "Sepsis incidence and outcome: contrasting the intensive care unit with the hospital ward," Critical Care Medicine, vol. 35, no. 5, pp. 1284-1289, 2007.

[7] R. Cirocchi, A. Birindelli, W. Biffl et al., "What is the effectiveness of the negative pressure wound therapy (NPWT) in patients treated with open abdomen technique? A systematic review and meta-analysis," Journal of Trauma and Acute Care Surgery, vol. 81, no. 3, pp. 575-584.

[8] P. Navsaria, A. Nicol, D. Hudson, J. Cockwill, and J. Smith, "Negative pressure wound therapy management of the "open abdomen" following trauma: a prospective study and systematic review," World Journal of Emergency Surgery, vol. 8, no. 1, p. 4, 2013.

[9] O. Chiara, S. Cimbanassi, W. Biffl et al., "International consensus conference on open abdomen in trauma," Journal of Trauma and Acute Care Surgery, vol. 80, no. 1, pp. 173-183.

[10] R. R. Ivatury, "Update on open abdomen management: achievements and challenges," World Journal of Surgery, vol. 33, no. 6, pp. 1150-1153, 2009.

[11] F. A. Robledo, E. Luque-de-León, R. Suárez et al., "Open versus closed management of the abdomen in the surgical treatment of severe secondary peritonitis: a randomized clinical trial," Surgical Infections, vol. 8, no. 1, pp. 63-72, 2007. 
[12] S. Occhionorelli, M. Zese, L. Cappellari et al., "A life threatening case of perforated gangrenous appendicitis: when the open abdomen technique can be safe and effective in acute peritonitis. A Case Report," JSM Gastroenterol Hepatol, vol. 4, no. 3, p. 1061.

[13] A. J. McCosh II, "The treatment of general septic peritonitis," Annals of Surgery, vol. 25, no. 6, pp. 687-697, 1897.

[14] R. P. Dellinger, M. M. Levy, J. M. Carlet et al., "American Association of Critical-Care Nurses; American College of Chest Physicians; American College of Emergency Physicians; Canadian Critical Care Society; European Society of Clinical Microbiology and Infectious Diseases; European Society of Intensive Care Medicine; European Respiratory Society; International Sepsis Forum; Japanese Association for Acute Medicine; Japanese Society of Intensive Care Medicine; Society of Critical Care Medicine; Society of Hospital Medicine; Surgical Infection Society; World Federation of Societies of Intensive and Critical Care Medicine. Surviving sepsis campaign: international guidelines for management of severe sepsis and septic shock: 2008," Critical Care Medicine, vol. 36, no. 1, pp. 296-327, 2008.

[15] M. Sartelli, F. Catena, L. Ansaloni et al., "Complicated intraabdominal infections worldwide: the definitive data of the CIAOW study," World Journal of Emergency Surgery, vol. 9, no. 1, p. 37, 2014

[16] Y. Chen, J. Ye, W. Song, J. Chen, Y. Yuan, and J. Ren, "Comparison of outcomes between early fascial closure and delayed abdominal closure in patients with open abdomen: a systematic review and meta-analysis," Gastroenterology Research and Practice, vol. 2014, Article ID 784056, 8 pages, 2014.

[17] C. A. Ordonez, A. I. Sanchez, J. A. Pineda et al., "Deferred primary anastomosis versus diversion in patients with severe secondary peritonitis managed with staged laparotomies," World Journal of Surgery, vol. 34, no. 1, pp. 169-176, 2010.

[18] J. S. Paul and T. J. Ridolfi, "A case study in intra-abdominal sepsis," The Surgical Clinics of North America, vol. 92, no. 6, pp. 1661-1677, 2012.

[19] A. Lambertz, C. Mihatsch, A. Röth et al., "Fascial closure after open abdomen: initial indication and early revisions are decisive factors - a retrospective cohort study," International Journal of Surgery, vol. 13, pp. 12-16, 2015.

[20] S. K. Rasilainen, M. P. Juhani, and A. Leppäniemi, "Microbial colonization of open abdomen in critically ill surgical patients," World Journal of Emergency Surgery, vol. 10, p. 25, 2015.

[21] J. J. De Waele, “Abdominal sepsis," Current Infectious Disease Reports, vol. 18, no. 8, p. 23, 2016.

[22] A. Leppäniemi, E. J. Kimball, I. De Iaet, M. L. Malbrain, Z. J. Balogh, and J. J. De Waele, "Management of abdominal sepsis - a paradigm shift?," Anaesthesiology Intensive Therapy, vol. 47, no. 4, pp. 400-408, 2015.

[23] G. Leroy, F. Lambiotte, G. Thévenin et al., "Evaluation of "Candida score" in critically ill patients: a prospective, multicenter, observational, cohort study," Annals of Intensive Care, vol. 1, no. 1, p. 50, 2011.

[24] O. Marchetti, J. Bille, U. Fluckiger et al., "Epidemiology of candidemia in Swiss tertiary care hospitals: secular trends, 1991-2000," Clinical Infectious Diseases, vol. 38, no. 3, pp. 311-320, 2004.

[25] D. H. Kett, E. Azoulay, P. M. Echeverria, J. L. Vincent, and Extended Prevalence of Infection in ICU Study (EPIC II) Group of Investigators, "Candida bloodstream infections in intensive care units: analysis of the extended prevalence of infection in intensive care unit study," Critical Care Medicine, vol. 39, no. 4, pp. 665-670, 2011.

[26] P. Eggimann, Y. A. Que, J. P. Revelly, and J. L. Pagani, "Preventing invasive Candida infections. Where could we do better?," Journal of Hospital Infection, vol. 89, no. 4, pp. 302308, 2015.

[27] J. L. Vincent, R. Moreno, J. Takala et al., "The SOFA (sepsisrelated organ failure assessment) score to describe organ dysfunction/failure," Intensive Care Medicine, vol. 22, no. 7, pp. 707-710, 1996.

[28] A. E. Jones, S. Trzeciak, and J. A. Kline, “The sequential organ failure assessment score for predicting outcome in patients with severe sepsis and evidence of hypoperfusion at the time of emergency department presentation," Critical Care Medicine, vol. 37, no. 5, pp. 1649-1654, 2009.

[29] N. Shapiro, M. D. Howell, D. W. Bates, D. C. Angus, L. Ngo, and D. Talmor, "The association of sepsis syndrome and organ dysfunction with mortality in emergency department patients with suspected infection," Annals of Emergency Medicine, vol. 48, no. 5, pp. 583-590.e1, 2006.

[30] M. Björck, A. W. Kirkpatrick, M. Cheatham, M. Kaplan, A. Leppäniemi, and J. J. de Waele, "Amended classification of the open abdomen," Scandinavian Journal of Surgery, vol. 105, no. 1, pp. 5-10, 2016.

[31] D. Dindo, N. Demartines, and P. A. Clavien, "Classification of surgical complications. A new proposal with evaluation in a cohort of 6336 patients and results of a survey," Annals of Surgery, vol. 240, no. 2, 2004.

[32] S. Baker and B. O’Neill, "The injury severity score. An update," The Journal of Trauma, vol. 16, no. 1.

[33] American Society of Anesthesiologists Physical Status Classification System, ASA House of Delegates, October 2014, http:// www.asahq.org/resources/clinical-information/asa-physicalstatus-classification-system.

[34] A. W. Kirkpatrick, D. J. Roberts, J. De Waele et al., "Intraabdominal hypertension and the abdominal compartment syndrome: updated consensus definitions and clinical practice guidelines from the World Society of the Abdominal Compartment Syndrome," Intensive Care Medicine, vol. 39, no. 7, pp. 1190-1206, 2013.

[35] Sartelli et al., "Advantages and disadvantages of different types of temporary abdominal closure (TAC) techniques," World Journal of Emergency Surgery, vol. 10, p. 35, 2015.

[36] A. T. Cristaudo, S. B. Jennings, K. Hitos, R. Gunnarsson, and A. DeCosta, "Treatments and other prognostic factors in the management of the open abdomen: a systematic review," Journal of Trauma and Acute Care Surgery, vol. 82, no. 2, pp. 407-418.

[37] F. Coccolini, W. Biffl, F. Catena et al., "The open abdomen, indications, management and definitive closure," World Journal of Emergency Surgery, vol. 10, no. 1, p. 32, 2015.

[38] M. D. Parkins, D. M. Sabuda, S. Elsayed, and K. B. Laupland, "Adequacy of empirical antifungal therapy and effect on outcome among patients with invasive Candida species infections," The Journal of Antimicrobial Chemotherapy, vol. 60, no. 3, pp. 613-618, 2007.

[39] N. I. Paphitou, L. Ostrosky-Zeichner, and J. H. Rex, "Rules for identifying patients at increased risk for candidal infections in the surgical intensive care unit: an approach to developing practical criteria for systematic use in antifungal 
prophylaxis trials," Medical Mycology, vol. 43, no. 3, pp. 235243, 2005.

[40] A. F. Shorr, Y. P. Tabak, R. S. Johannes, X. Sun, J. Spalding, and M. H. Kollef, "Candidemia on presentation to the hospital: development and validation of a risk score," Critical Care, vol. 13, no. 5, p. R156, 2009.

[41] C. Leon, S. Ruiz-Santana, P. Saavedra et al., "A bedside scoring system ("Candida score") for early antifungal treatment in nonneutropenic critically ill patients with Candida colonization*," Critical Care Medicine, vol. 34, no. 3, pp. 730-737, 2006.

[42] R. Goldberg, J. Henning, L. G. Wolfe, and T. M. Duane, "Practice patterns for the use of antibiotic agents in damage control laparotomy and its impact ion outcomes," Surgical Infections, vol. 18, no. 3, 2017.

[43] P. Eggimann, P. Francioli, J. Bille et al., "Fluconazole prophylaxis prevents intra-abdominal candidiasis in high-risk surgical patients," Critical Care Medicine, vol. 27, no. 6, pp. 1066-1072, 1999.

[44] F. Coccolini, G. Montori, M. Ceresoli et al., "IROA: IROA: international register of open abdomen, preliminary results," World Journal of Emergency Surgery, vol. 12, p. 10, 2017.

[45] F. Coccolini, F. Catena, G. Montori et al., "IROA: The International Register of Open Abdomen. An international effort to better understand the open abdomen: call for participants," World Journal of Emergency Surgery, vol. 10, p. 37, 2015.

[46] F. Coccolini, G. Montori, M. Ceresoli et al., "The role of open abdomen in non-trauma patient: WSES consensus paper," World Journal of Emergency Surgery, vol. 12, p. 39, 2017.

[47] V. Rodolico, P. di Carlo, G. Gulotta et al., "Intra-abdominal Candida spp infection in the acute abdomen in a quality assurance (QA)-certified academic setting," Journal of Clinical Pathology, vol. 70, no. 7, pp. 579-583, 2017.

[48] G. Gökahmetoğlu, F. Mutlu Sarıüzel, A. N. Koç et al., "Determination of Candida colonization and Candida score in patients in anesthesia intensive care unit," Mikrobiyoloji Bülteni, vol. 50, no. 3, pp. 438-448, 2016. 


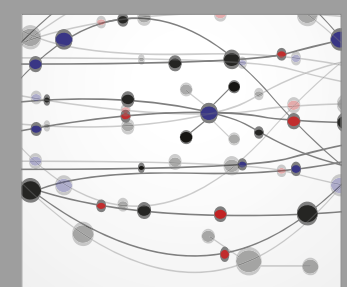

The Scientific World Journal
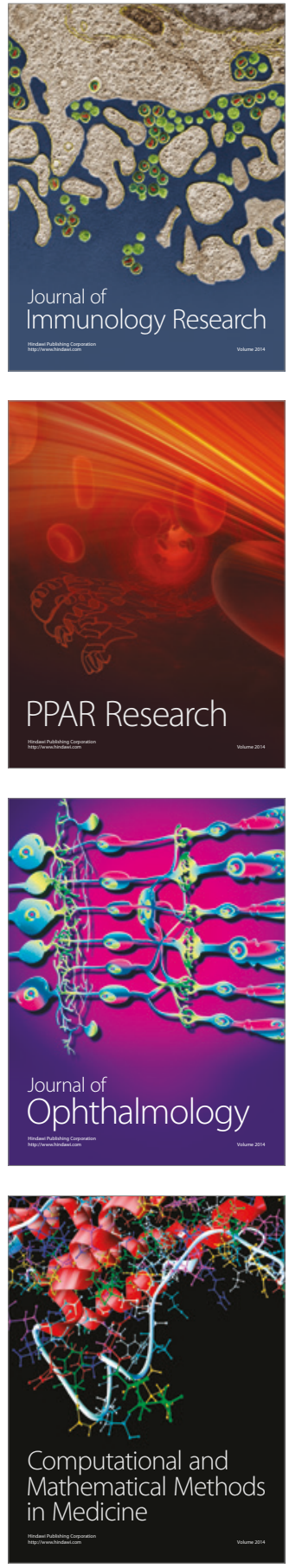

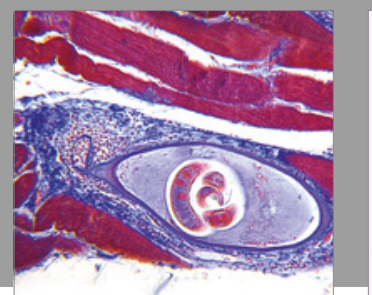

Gastroenterology Research and Practice
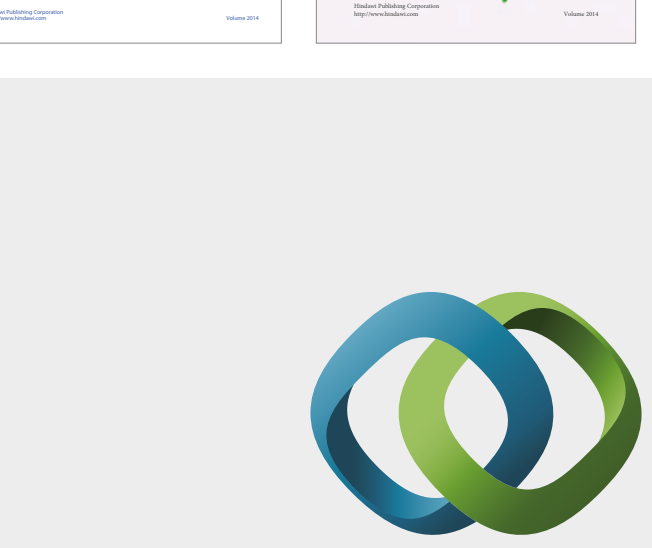

\section{Hindawi}

Submit your manuscripts at

https://www.hindawi.com
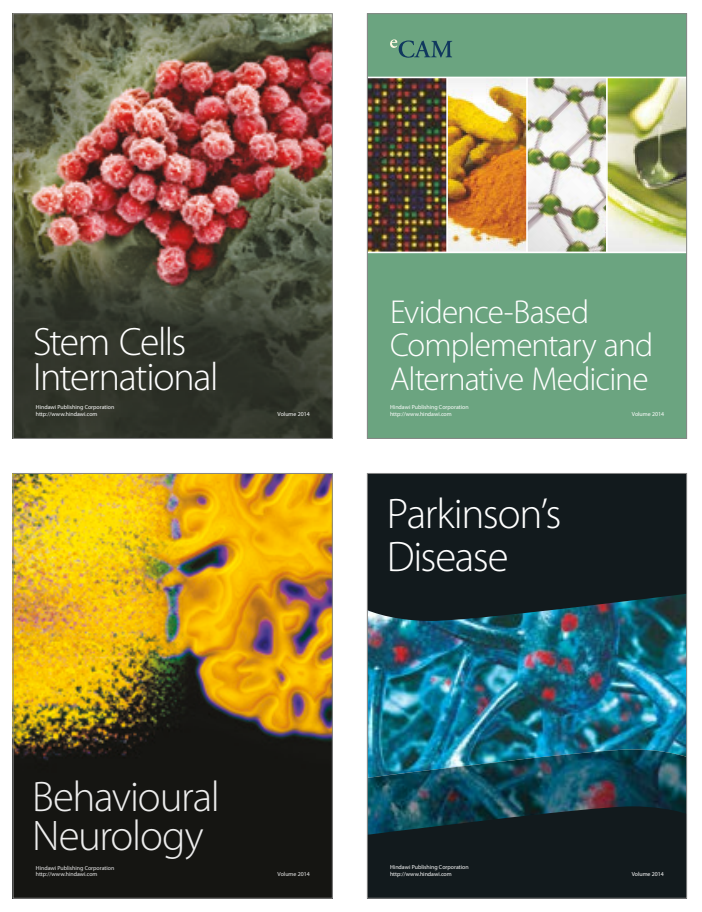
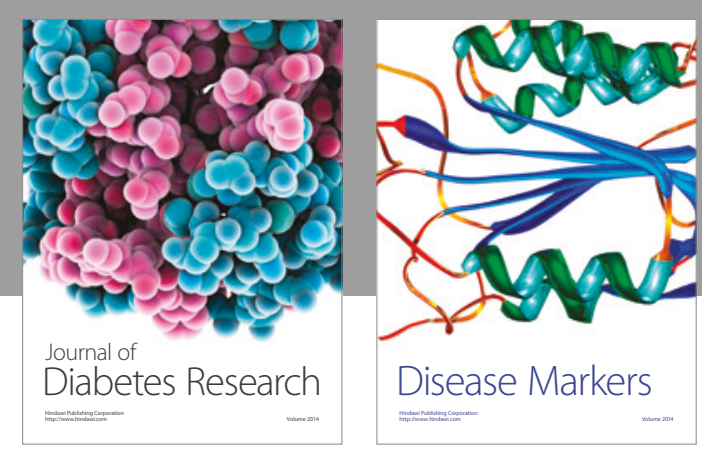

Disease Markers
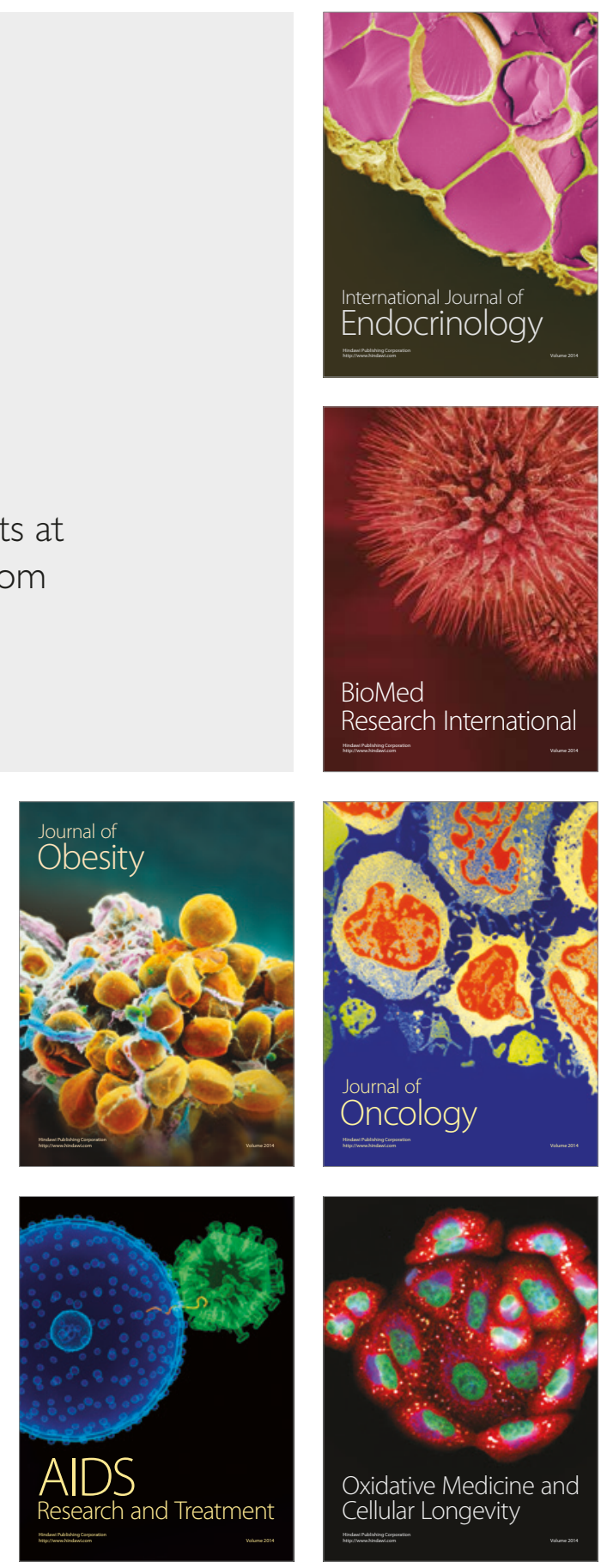